\title{
A Japanese adult and two girls with NEDMIAL caused by de novo missense variants in $D H X 30$
}

\author{
Kimiko Ueda ${ }^{1 凶}$, Atsushi Araki ${ }^{2}$, Atsushi Fujita ${ }^{3}$, Naomichi Matsumoto ${ }^{3}$, Tomoko Uehara ${ }^{4,5}$, Hisato Suzuki ${ }^{4}$, Toshiki Takenouchi ${ }^{6}{ }^{6}$, \\ Kenjiro Kosaki ${ }^{4}$ and Nobuhiko Okamoto ${ }^{1}{ }^{1}$
}

(c) The Author(s) 2021

Lessel et al. reported a novel neurodevelopmental disorder with severe motor impairment and absent language (NEDMIAL) in 12 individuals and identified six different de novo heterozygous missense variants in $D H X 30$. The other clinical features included muscular hypotonia, feeding difficulties, brain anomalies, autistic features, sleep disturbances, and joint hypermobility. We report a Japanese adult with a novel missense variant and two girls with de novo missense variants in $D H X 30$.

Human Genome Variation (2021) 8:1-4; https://doi.org/10.1038/s41439-021-00155-9

DHX30 is a member of the DEAH-box family of RNA helicases. DHX30 is located on chromosome 3p21.31, and it encodes an ATPdependent RNA helicase. The homozygous deletion of DHX30 leads to early embryonic lethality and causes early developmental defects in the central nervous system in mice ${ }^{1}$. Lessel et al. reported a novel neurodevelopmental disorder with severe motor impairment and absent language (NEDMIAL; MIM \#617804) in 12 individuals and identified six different de novo heterozygous missense variants in the $D H X 30$ gene $^{2}$. Recently, two brothers with the same de novo missense variation in $D H X 30$ by gonadal mosaicism were reported ${ }^{3}$.

NEDMIAL is characterized by severely delayed psychomotor development, which causes an autosomal dominant disorder. The affected patients show muscular hypotonia, feeding difficulties, ataxic gait, or an inability to walk. Their cognitive development is severely impaired. Speech development is minimal or absent, and severe intellectual disability is a constant feature. It is also characterized by behavioral abnormalities, such as autistic features, low frustration tolerance, hand-flapping, and stereotypies. Additional common features may include sleep disorders, nonspecific dysmorphic facial features, and joint hypermobility.

We report herein a Japanese adult and two girls with de novo missense $D H X 30$ variants.

Patient 1 was a 22-year-old male who was the first child of healthy and nonconsanguineous Japanese parents. His birth weight was $2864 \mathrm{~g}(-1.3$ standard deviations (SD)), length was $49 \mathrm{~cm}(-0.7 \mathrm{SD})$, and head circumference was $32 \mathrm{~cm}(-1.5 \mathrm{SD})$ at 41 weeks of gestation. He showed severe global developmental delay from the early infantile period. He was referred to the city hospital at 2 years of age, and a magnetic resonance imaging (MRI) brain scan indicated brain atrophy. Complex partial seizures were noted for the first time at age 3 and did not occur again subsequently, although electroencephalography revealed multifocal epileptic spikes. Puberty was delayed, and changes in his voice occurred after he was 20 years old. He gradually developed a Rett syndrome-like presentation. His karyotype by G-banding analysis was 46, XY. Rett syndrome and Angelman syndrome were excluded by molecular analysis. He was referred to our center for detailed examination. His height was $149 \mathrm{~cm}$ (-3.3 SD), weight

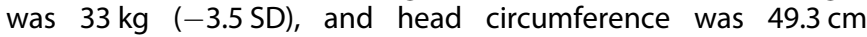
$(-0.6 \mathrm{SD})$ at 22 years of age. He was bedridden and could still not crawl or stand, although he could sit at the age of 3 . He had scoliosis and bilateral hip dislocations. He also showed periodic hyperventilation, frequent bouts of laughing, and sleep disturbance. He showed all emotions without words and could not understand simple commands. He needed careful supervision and help in all of his daily activities.

Patient 2 was a 7-year-old girl who was the first child of healthy and nonconsanguineous Japanese parents. Her birth weight was $3258 \mathrm{~g}(-1.2 \mathrm{SD})$, length was $48 \mathrm{~cm}(+0.3 \mathrm{SD})$, and head circumference was $33.5 \mathrm{~cm}(-0.2 \mathrm{SD})$ at 41 weeks of gestation. She was hypotonic, and motor development was delayed. She could roll over at 12 months of age, crawl at 3 years of age, and sit at 4 years of age. Her expressive language was severely delayed. She gradually developed a Rett syndrome-like presentation. However, no variants or deletions were found in methyl-CpG-binding protein $2(M E C P 2)^{4}$. She did not experience epileptic seizures. Physical examination revealed dysmorphic features, including thick low-set ears, long eyelashes, interdental space, a high arched palate, and micrognathia (Fig. 1a). Her hands and feet were small (Fig. 1b). Her height was $103 \mathrm{~cm}$ (-3.2 SD), weight was $14 \mathrm{~kg}$ $(-2.4 \mathrm{SD})$, and head circumference was $47.1 \mathrm{~cm}(-3.1 \mathrm{SD})$. She could not stand or speak at 7 years of age. Brain MRI showed a decreased white matter volume and enlarged brain ventricles and extra-axial space (Fig. 1e). Electroencephalography revealed multifocal spike discharges. Conventional cytogenetic studies, array comparative genomic hybridization, and investigations for metabolic abnormalities yielded normal results.

\footnotetext{
${ }^{1}$ Department of Medical Genetics, Osaka Women's and Children's Hospital, Izumi, Osaka, Japan. ${ }^{2}$ Nakano Children's Hospital, Osaka, Japan. ${ }^{3}$ Department of Human Genetics, Yokohama City University Graduate School of Medicine, Yokohama, Japan. ${ }^{4}$ Center for Medical Genetics, Keio University School of Medicine, Tokyo, Japan. ${ }^{5}$ Department of
}

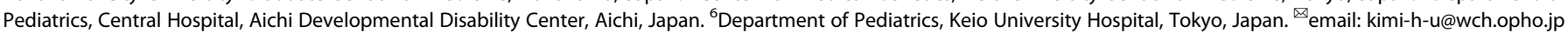




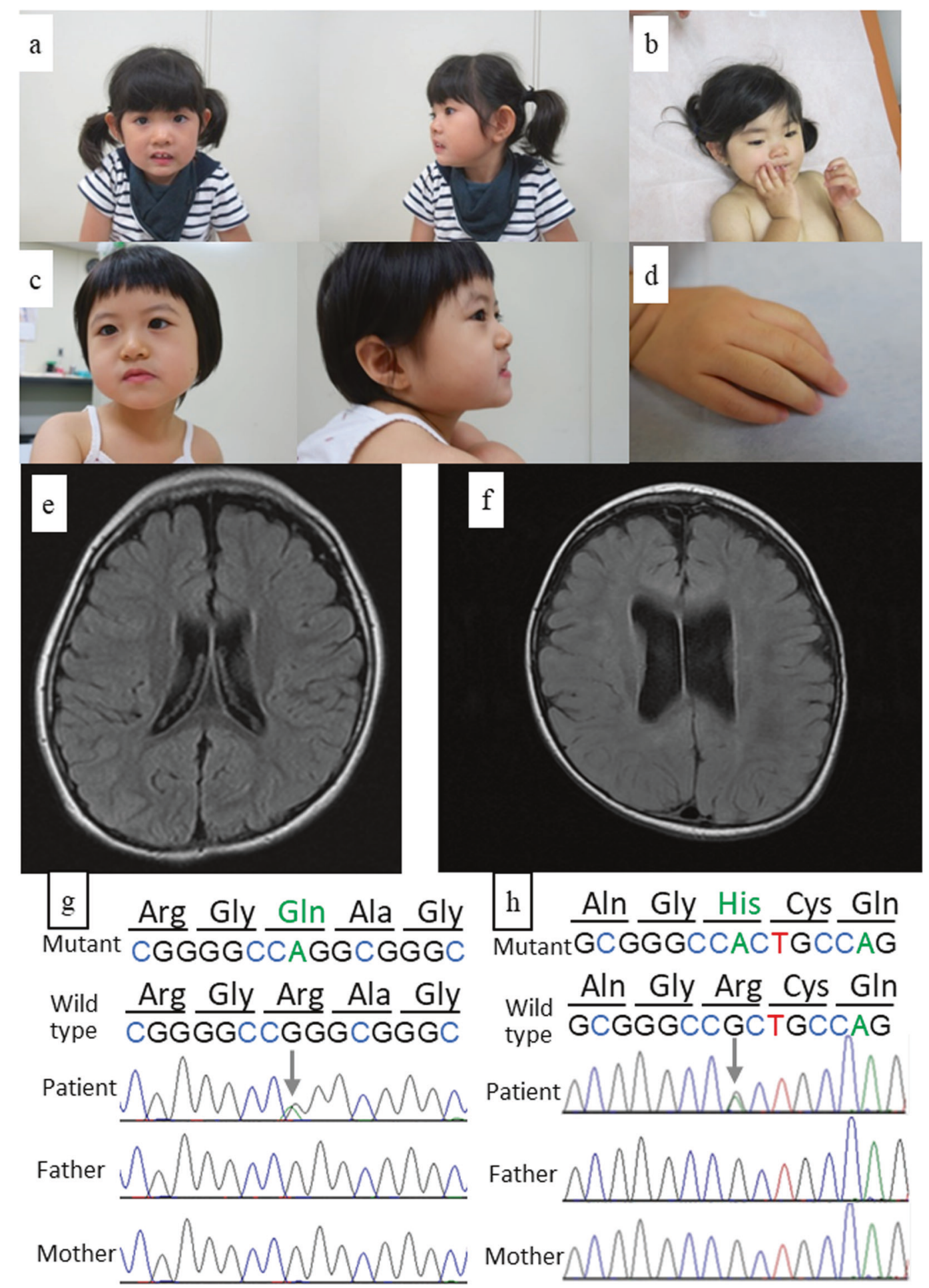

Fig. 1 Patients with DHX30 variants and their MRI findings. a Patient 2 at 3 years of age. The patient exhibited thick low-set ears, long eyelashes, interdental space, high arched palate, micrognathia, b tapered fingers and hand licking. $\mathbf{c}$ Patient 3 at 5 years of age. The patient exhibited internal strabismus, epicanthus, thick eye brow, long eyelashes, and $\mathbf{d}$ tapered fingers. e Axial T1-weighted brain MRI of Patient 2 demonstrated a decreased white matter volume and enlarged brain ventricles and extra-axial space. f Axial T2-weighted fluid-attenuated inversion recovery brain MRI of Patient 3 demonstrated significant decreases in the volume of the midbrain and ventricular dilatation and completed myelination with remaining multiple areas of patchy increased T2 signal around the cerebral white matter. DHX30 variants were identified in patients 1 (g) and 2 (h) by the Sanger method. Arrows indicate variations.

Patient 3 was a 5 -year-old girl who was the second child of healthy and nonconsanguineous Japanese parents. She had an unaffected older sister. Her birth weight was $2556 \mathrm{~g}(-0.9 \mathrm{SD})$, length was $46 \mathrm{~cm}(-1.1 \mathrm{SD})$, and head circumference was $32.4 \mathrm{~cm}$
$(-0.5 \mathrm{SD})$ at 38 weeks of gestation. She suckled at the breast well. However, she could not eat well after starting baby food. Delayed neck control was noted at 5 months of age. She was referred to a city hospital at 11 months of age, and an MRI brain scan indicated 
brain atrophy. She could roll over at 7 months of age, control her head at 12 months of age, and sit at 24 months of age. She was diagnosed by whole exome sequencing (WES) in the United States of America, where she moved at 26 months of age. After she returned to Japan, she visited our center at 5 years of age. Her height was $95.1 \mathrm{~cm}(-3.1 \mathrm{SD})$, weight was $10.9 \mathrm{~kg}(-3.1 \mathrm{SD})$, and head circumference was $46.4 \mathrm{~cm}(-2.4 \mathrm{SD})$. She showed muscular hypotonia and moved only by rolling over. She had feeding difficulties. Her pathognomonic behaviors were hand-licking, bruxism, a happy demeanor, such as frequent smiling and laughter, and a low frustration tolerance. Physical examination revealed dysmorphic features, including internal strabismus, epicanthus, thick eyebrows and long eyelashes, and a high arched palate, tapered fingers, joint hypermobility, and overlapping toes (Fig. 1c, d). She suffered from sleep disorder. She did not experience seizures, although electroencephalography revealed abnormalities in the awake and sleep states due to mild slowing and disorganization for her age. Brain MRI at 3 years of age revealed significant decreases in the volume of the midbrain and ventricular dilatation and arachnoid cysts in the middle cranial fossa; in addition, it showed completed myelination with remaining multiple areas of patchy increased T2 signal around the cerebral white matter (Fig. 1f). Swallowing videofluorography, electromyography, and nerve conduction velocity were normal.

With the approval of our institutional ethics committees, blood samples were collected from each participating individual, and after genomic DNA was extracted, the patients underwent WES. The pathogenicity of the variants was evaluated according to the American College of Medical Genetics and Genomics (ACMG) variant interpretation guidelines. In patient 1 , a de novo missense variant in DHX30 (NM_138615.2), c.2345 G>A, p. (Arg782Gln), was revealed (Fig. 1g). The variant was novel, although it was located just next to c.2344 C>T, p.(Arg782Trp), which was reported by Lessel et al. ${ }^{2}$. This variant was judged to be likely pathogenic according to the ACMG guidelines. The variant was predicted to be likely pathogenic by a SIFT score of 0 , PolyPhen2 score of 1 , and CADD score of 33 . In patient 2 , a de novo missense variant in DHX30, c.2354 G>A, p.(Arg785His), was revealed (Fig. $1 \mathrm{~h}$ ). This variant was previously reported by Lessel et al. ${ }^{2}$. In patient 3 , a de novo missense variant in $D H X 30$, c.2353 C>T, p.(Arg785Cys), which was also reported by Lessel et al. ${ }^{2}$, was revealed. All of the variants in our patients were in motif $\mathrm{VI}$ of the helicase core region of $D H X 30$, the nucleotideinteracting motif. All protein variants in ATP-binding motif VI showed obviously reduced ATPase activities, which provided strong evidence for the pathogenicity of NEDMIAL ${ }^{2}$. Our findings could add to this evidence.

We reported three patients with NEDMIAL. Patient 1 is the first adult patient to be reported based on diagnosis with genetic testing for the first time in adulthood, even though he has been affected with NEDMIAL since early childhood, and he had a novel variant in $D H X 30$.

Rett syndrome-like or Angelman syndrome-like features were noted in the three patients. Rett syndrome is a neurodevelopmental disorder mostly caused by variants in MECP2 ${ }^{4}$. However, variants in various other genes might lead to Rett syndrome-like phenotypes. Without WES, it is difficult to diagnose NEDMIAL. NEDMIAL should be considered in the differential diagnosis of Rett syndrome and Angelman syndrome.

Table 1. Clinical characteristics of our patients and the previously reported patients with $D H X 30$ alterations.

\begin{tabular}{|c|c|c|c|c|}
\hline Clinical findings & Patient 1 & Patient 2 & Patient 3 & $\begin{array}{l}14 \text { previously reported patients (Lessel } \\
\text { et al. }{ }^{2} \text {, Cross et al. } .^{3} \text { ) }\end{array}$ \\
\hline Sex & Male & Female & Female & 6 males, 8 females \\
\hline Age at last examination & 22 years & 7 years & 5 years & 3-17 years, 6 months, 15 months \\
\hline Intellectual disability & + & + & + & $14 / 14$ \\
\hline Speech ability & Non-verbal & Non-verbal & Non-verbal & 20 words, 4 words, 11 non-verbal, 1 N/A \\
\hline Motor development delay & + & + & + & $14 / 14$ \\
\hline Muscular hypotonia & + & + & + & $14 / 14$ \\
\hline Age at walking (years) & - & - & - & $28 / 12,6,3,5,8,11$ no walking, 1 N/A \\
\hline Gait abnormalities & $\begin{array}{l}\text { No independent } \\
\text { walking }\end{array}$ & $\begin{array}{l}\text { No independent } \\
\text { walking }\end{array}$ & $\begin{array}{l}\text { No independent } \\
\text { walking }\end{array}$ & 6 ataxic, 6 no independent walking, 2 N/A \\
\hline Autistic features & + & + & + & $+(7 / 12), 2 \mathrm{~N} / \mathrm{A}$ \\
\hline $\begin{array}{l}\text { No purposeful movement } \\
\text { of hands }\end{array}$ & + & + & + & $+(5 / 12), 2 \mathrm{~N} / \mathrm{A}$ \\
\hline Bruxism & - & + & + & $+(1 / 12), 2 \mathrm{~N} / \mathrm{A}$ \\
\hline Sleep disturbance & + & - & + & $+(7 / 14)$ \\
\hline Seizure & - & + & + & $+(3 / 14)$ \\
\hline Feeding difficulties & - & - & + & $+(11 / 14)$ \\
\hline Strabismus & - & - & + & $+(6 / 14)$ \\
\hline Joint hypermobility & + & - & + & $+(6 / 14)$ \\
\hline Unilateral cryptorchidism & - & $\mathrm{N} / \mathrm{A}$ & $\mathrm{N} / \mathrm{A}$ & $+(4 / 6)$ \\
\hline Brain MRI anomalies & + & + & + & $+(10 / 14)$ \\
\hline DHX30 alteration & $\begin{array}{l}\text { c. } 2345 \mathrm{G}>\mathrm{A}, \\
\text { p.(Arg } 782 \mathrm{Gln})\end{array}$ & $\begin{array}{l}\text { c. } 2354 \mathrm{G}>\mathrm{A}, \\
\text { p.(Arg } 785 \text { His) }\end{array}$ & $\begin{array}{l}\text { c. } 2353 \text { C>T, } \\
\text { p.(Arg785Cys) }\end{array}$ & $\begin{array}{l}\text { c. } 1478 \mathrm{G}>\mathrm{A}, \text { p.(Arg493His) (2/14), c. } 1685 \mathrm{~A}>\mathrm{G} \text {, } \\
\text { p.(His562Arg) (1/14), c.2093 C>T, p.(Ser698Phe) } \\
\text { (2/14) c.2342 G>A, p.(Gly781Asp) ( } 2 / 14), \\
\text { c. } 2344 \mathrm{C}>\mathrm{T} \text {, p.(Arg782Trp) (3/14), c. } 2353 \mathrm{C}>\mathrm{T}, \\
\text { p.(Arg785Cys) (3/14), c.2354 G>A, } \\
\text { p.(Arg785His) (1/14) }\end{array}$ \\
\hline
\end{tabular}

+ present, - absent, N/A not applicable. 
Brain MRI anomalies, including delayed myelination, cerebellar atrophy, dilated ventricles, cortical atrophy, and corpus callosal abnormalities, have been reported ${ }^{2}$. Our patients showed similar conditions. DHX30 is a member of the DEAH-box family of RNA helicases, and it is considered to be involved in several phases of the RNA lifecycle, leading to the initiation of mRNA translation ${ }^{5}$. Decreases in the functional level of DHX30 due to variants may lead to the progression of early developmental disorders ${ }^{6}$. DHX30 might play important roles in cerebral maturation, and brain MRI appears useful for the diagnosis of NEDMIAL.

In conclusion, we report a Japanese adult and two girls with NEDMIAL caused by missense variants in DHX30. We suggest that NEDMIAL is a novel neurodevelopmental disorder that may mimic Rett syndrome or Angelman syndrome in its early stage, and brain MRI anomalies are pathognomonic findings for the diagnosis of NEDMIAL (Table 1).

\section{HGV DATABASE}

The relevant data from this Data Report are hosted at the Human Genome Variation Database at https://doi.org/10.6084/m9. figshare.hgv.3024; https://doi.org/10.6084/m9.figshare.hgv.3027; https://doi.org/10.6084/m9.figshare.hgv.3030.

\section{REFERENCES}

1. Zheng, H. J. et al. The novel helicase helG (DHX30) is expressed during gastrulation in mice and has a structure similar to a human DExH box helicase. Stem Cells Dev. 24, 372-383 (2015).

2. Lessel, D. et al. De novo missense variants in DHX30 impair global translation and cause a neurodevelopmental disorder. Am. J. Hum. Genet 101, 716-724 (2017).

3. Cross, L. A., McWalter, K., Keller-Ramey, J., Henderson, L. B. \& Amudhavalli, S. M. A report of gonadal mosaicism in DHX30-related neurodevelopmental disorder. Clin. Dysmorphol. 29, 161-164 (2020)

4. Amir, R. E. et al. Rett syndrome is caused by variants in X-linked MECP2, encoding methyl-CpG-binding protein 2. Nat. Genet 23, 185-188 (1999).

5. Antonicka, H. \& Shoubridge, E. A. Cell Rep. Mitochondrial RNA granules are centers for posttranscriptional RNA processing and ribosome biogenesis. Cell Rep. 10, 920-932 (2015).
6. Holt, C. E., Martin, K. C. \& Schuman, E. M. Local translation in neurons: visualization and function. Nat. Struct. Mol. Biol. 26, 557-566 (2019).

\section{ACKNOWLEDGEMENTS}

This study was supported by the initiative on rare and undiagnosed diseases run by the Japan Agency for Medical Research and Development. This study was supported by the initiative on rare and undiagnosed diseases run by the Japan Agency for Medical Research and Development.

\section{CONFLICT OF INTEREST}

The authors declare no competing interests.

\section{ADDITIONAL INFORMATION}

Correspondence and requests for materials should be addressed to K.U.

Reprints and permission information is available at http://www.nature.com/ reprints

Publisher's note Springer Nature remains neutral with regard to jurisdictional claims in published maps and institutional affiliations. (c) Open Access This article is licensed under a Creative Commons adaptation, distribution and reproduction in any medium or format, as long as you give appropriate credit to the original author(s) and the source, provide a link to the Creative Commons license, and indicate if changes were made. The images or other third party material in this article are included in the article's Creative Commons license, unless indicated otherwise in a credit line to the material. If material is not included in the article's Creative Commons license and your intended use is not permitted by statutory regulation or exceeds the permitted use, you will need to obtain permission directly from the copyright holder. To view a copy of this license, visit http://creativecommons. org/licenses/by/4.0/.

(c) The Author(s) 2021 